\title{
Option bei schwerer Anorexia nervosa?
}

Fragestellung: Ist die tiefe Hirnstimulation (THS) ein wirksamer therapeutischer Ansatz bei Patientinnen mit schweren Verlaufsformen einer Anorexia nervosa?

Hintergrund: Die THS ist bei einer Reihe von neuropsychiatrischen Erkrankungen, bei denen dysfunktionale neuronale Netzwerke identifiziert wurden, erfolgreich eingesetzt worden. Diese Arbeit prüfte, inwieweit die Modulation von limbischen Strukturen mithilfe dieses neurochirurgischen Verfahrens den klinischen Verlauf der Anorexia nervosa beeinflussen kann.

Patienten und Methodik: Um dies zu überprüfen sind in einer prospektiven Phase-I-Studie sechs Patientinnen mit schwerer, therapierefraktärer oder chronischer Anorexia nervosa im Alter zwischen 20 und 60 Jahren mit THS des subgenualen Cingulum behandelt worden (die individuellen Elektrodenpositionierungen sind in $\triangleright A b b .1$ dargestellt). Body-Mass-Index (BMI), Psychometrie sowie bildgebende Verfahren wurden vor Beginn der Therapie und zu weiteren definierten Zeitpunkten in einem Zeitraum von neun Monaten nach der Operation durchgeführt.

Ergebnisse: Die THS ging mit einigen unerwünschten Wirkungen einher; eine Patientin erlitt einen epileptischen Anfall. Nach neun Monaten hatten drei der sechs Patienten einen BMI der hö-

Lipsman N, Woodside DB, Giacobbe $P$ et al. Subcallosal cingulate deep brain stimulation for treatment-refractory anorexia nervosa: a phase 1 pilot trial. Lancet 2013; publ online: doi. org/10.1016/S0140-6736 (12) $62188-6$ her als der historische Tiefstwert war. Nach sechs Mona-
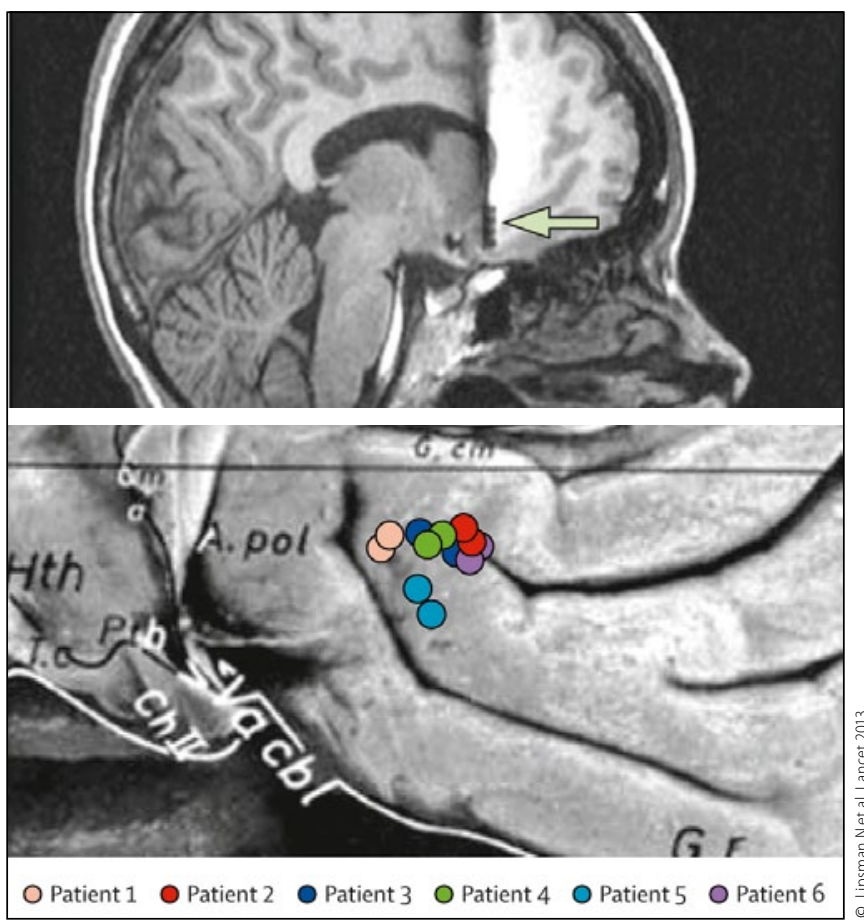

1 Positionierung der Elektroden bei den einzelnen Patientinnen. ten konnten bei vier Patientinnen Verbesserungen bei Stimmung, Angstsymptomen, Affektregulation und anorexieassoziierten Zwangssymptomen sowie bei drei Patientinnen Verbesserungen der Lebensqualität verzeich- net werden. PET-Untersuchungen zeigten, dass die klinischen Verbesserungen mit Aktivitätsänderungen im anterioren Cingulum, der Insula und dem parietalen Kortex einhergingen.

Schlussfolgerungen: Die THS stellt eine relativ sichere Behandlungsmethode bei Patientinnen mit therapierefraktärer Anorexia nervosa dar.

\section{Vom klinischen Alltag noch weit entfernt}

In dieser Pilotstudie wurden erstmalig sechs Patientinnen mit schweren, therapieresistenten oder chronischen Essstörungen mit tiefer Hirnstimulation behandelt. Interessant ist diese Studie, da hier das Indikationsgebiet der tiefen Hirnstimulation auf eine neue psychiatrische Indikation ausgeweitet wird. Das gewählte Studiendesign sah keine Randomisierung oder Verblindung vor, sodass diese klinische Interventionsstudie ein Punktwert von unter 3 auf der Jadad-Skala und nach üblichen EbM-Richtlinien lediglich ein Evidenzlevel von 4 erreicht. Diese Kenndaten sind nicht ungewöhnlich bei der Einführung neuer therapeutischer Interventionen, allerdings sollten zukünftige Therapien ausschließlich in placebokontrollierten und randomisierten Studien in ausgewählten spezialisierten Zentren durchgeführt werden. Zurzeit ist das Verfahren jedoch weit davon entfernt breit als therapeutische Alternative in der Behandlung von Essstörungen eingesetzt zu werden.

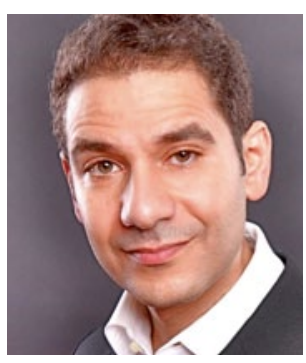

Prof. Dr. med. Malek Bajbouj, Berlin

Klinik für Psychiatrie (CBF), Charité, und Dahlem Institute for Neuroimaging of Emotion, Berlin

E-Mail: malek.bajbouj@charite.de 